\title{
PHOSPHATASE ACTIVITY IN THE OCULAR TISSUES*
}

\author{
BY \\ J. L. REIS \\ The Postgraduate Medical School of London
}

THE presence of phosphatases in the ocular tissues has been mentioned in several papers. Conciliis (1934) found alkaline phosphatase in the retina. Reis $(1937 a$ and $b ; 1940)$ found that in the retina and choroid of nearly all animals examined (frog, calf, rabbit, horse, dog, pig, but not pigeon) a specific phosphatase is much more active than the alkaline phosphatase.

This specific phosphatase is a 5-nucleotidase; it differs from the alkaline phosphatase in its range of action and in its optimal $p \mathrm{H}$. Whereas alkaline phosphatase, which is non-specific, hydrolyses a great variety of phosphoric esters, the 5-nucleotidase hydrolyses only nucleotides with the phosphate group attached to the fifth carbon of the pentose, that is, muscle adenylic acid (adenosine-5-monophosphoric acid) and inosinic acid (inosine-5-monophosphoric acid). The optimal $p \mathrm{H}$ of alkaline phosphatase is near $p \mathrm{H} \mathrm{9.5;} \mathrm{the} \mathrm{optimal} p \mathrm{H}$ of 5-nucleotidase is at $p \mathrm{H} 7.8$ (for human tissues), which approaches the physiological hydrogen ion concentration.

The activity of 5-nucleotidase varies in the eye tissues of different species. In calf and rabbit retina and choroid, at physiological $p \mathrm{H}$, the activity of 5-nucleotidase is about thirty times greater than that of the non-specific phosphatases (this means that the adenylic acid

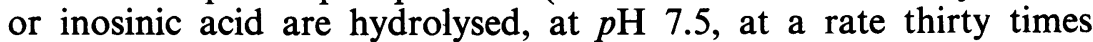
greater than any other phosphoric ester). The activity of 5 -nucleotidase in calf retina is about twice as great as in the choroid. The reverse was found for the rabbit, where the 5-nucleotidase activity in the choroid was sometimes four times greater than in the retina. Gulland and Jackson (1938) studied the 5-nucleotidase and non-specific phosphatase activity in the sheep's retina. They found a comparatively low activity, but even so, in the first hours of their experiments, the 5-nucleotidase activity was much more pronounced than that of the non-specific phosphatase.

Süllmann (1947) and Süllmann and Payot (1949), using a histochemical method, investigated the presence of alkaline phosphatase in animal cornea. They found this enzyme in epithelium, and in endothelium in some animals, but never in stroma. By a similar method, but using frozen sections, Friedenwald and Crowell (1949) studied the action of the alkaline phosphatases of the corneal epithelium upon the nucleic acids.

* Received for publication November 13, 1950. 
The activity of phosphatases in the human eye has not so far been studied, and the differences found between various species are too great to allow any inferences to be made by analogy. For this reason, some estimations on human eye tissues seemed desirable, and the following investigations were carried out with this object in view.

\section{METHOD}

The preparation of tissue extracts and the phosphatase-activity estimations were made as described in previous papers (Reis, 1937a and b; 1940; 1951). For the estimations of the non-specific phosphatases, sodium phenylphosphate was used as a substrate, as recommended by King and Armstrong (1934). Compared with the $\beta$-glycerophosphate, the phenylphosphate has a similar rate of hydrolysis at

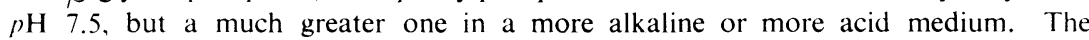
hydrolysis of phenylphosphate represents only the activity of the non-specific phosphatases, since it is not hydrolysed by the 5-nucleotidase. In order to estimate the specific phosphatase, that is the 5-nucleotidase, muscle adenylic acid was used. This ester is hydrolysed as well by the 5-nucleotidase as by the non-specific phosphatases. The difference between the rates of hydrolysis of adenylic acid and phenylphosphate represents the activity of 5-nucleotidase.

Human eyes for these estimations were obtained from the post-mortem room about 24 hours after death. Experiments on animal tissues had already shown that for the estimation of phosphatases fresh tissues are not essential.

\section{RESULTS}

Fig. 1 shows the activities of the phosphatases in human eye tissues. The most important are the activities near the physiological hydrogen ion concentration, $p \mathrm{H}$ 7.5. These estimations allow conclusions to be drawn regarding the physiological activity of the enzymes concerned. The non-specific phosphatases at $p \mathrm{H} 7.5$ have in all eye tissues a very low activity. In order to identify the nonspecific phosphatases, one estimation of phosphatase activity was performed at $p \mathrm{H} 9.0$ (for the alkaline phosphatase) and one at $p \mathrm{H} 5.5$ (for the acid phosphatase). As the acid phosphatase activity was found to be very low, nearly the whole non-specific phosphatase activity at physiological $p \mathrm{H}$ must therefore be ascribed to the alkaline phosphatase. The alkaline phosphatase was found to be exceptionally active in the choroid. The 5-nucleotidase is much more active in the retina and choroid than in the ciliary body and the iris. No evidence was found of any phosphatase activity in the sclera, cornea, lens, or vitreous body.

Fig. 2 shows the activities of phosphatases in the rabbit's eye. The enzyme activities are approximately similar to those in the human eye, except that the 5-nucleotidase activity in the choroid was found to be much higher in the rabbit $(6 \times)$.

The alkaline phosphatase of corneal epithelium described by Süllman and Payot (1949) could not be demonstrated in those experiments where an extract of the whole cornea was employed. 

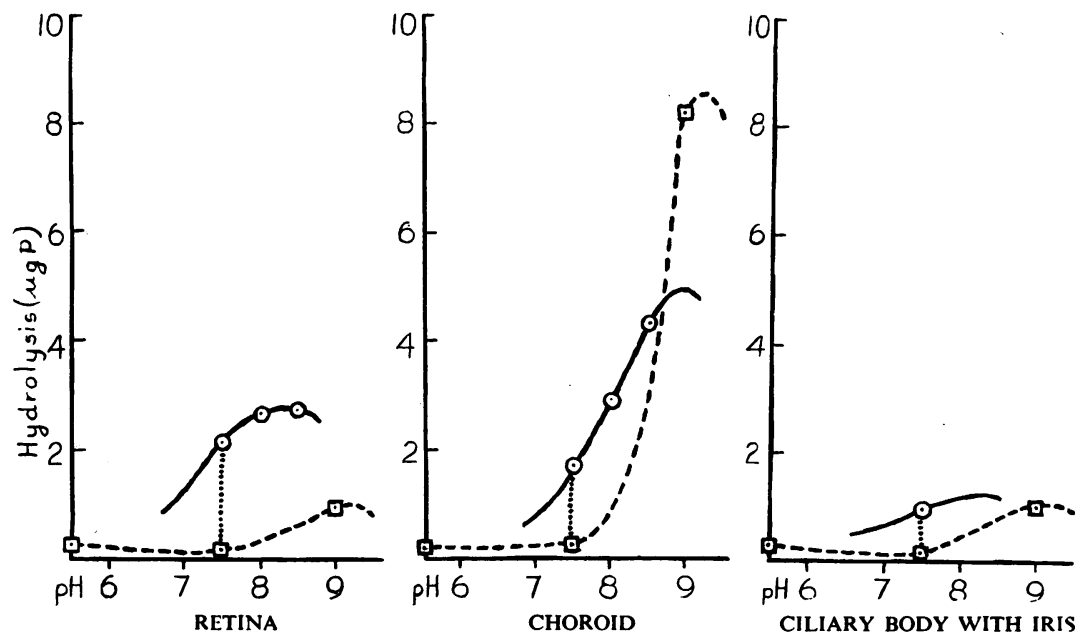

FIG. 1.-Activity of phosphatases in human intra-ocular tissues.

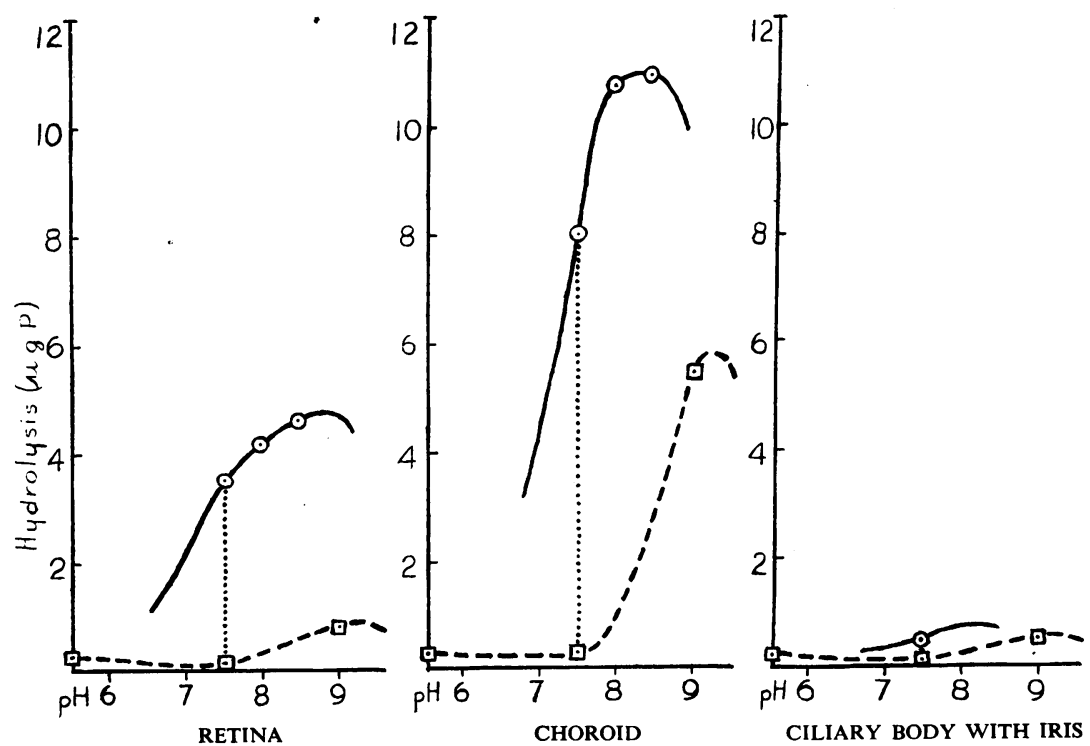

Fig. 2.-Activity of phosphatases in the intra-ocular tissues of the rabbit.

$$
\begin{gathered}
\text { Key to Figures: } \begin{array}{l}
\text { Amount of tissue: } 2 \mathrm{mg} . \\
\text { Incubation time }: 30 \mathrm{~min} . \\
\text { T.: } 38^{\circ} \text {. }
\end{array} \\
\text { Substrates }(0.0008 \mathrm{M}): \begin{array}{l}
- \\
-
\end{array}
\end{gathered}
$$

: : Hydrolysis due to action of 5-nucleotidase at physiological $\mathrm{FH}$ 
Probably the alkaline phosphatase of the epithelium was too diluted to be estimated by the chemical method used.

\section{Discussion}

The role played by the phosphatases in the eye, as in other tissues, is unknown. It is of interest that a high activity of the alkaline phosphatase may be found in those tissues where filtration is going on, e.g., kidney cortex, placenta, and choroid plexus. The activity of alkaline phosphatase in the choroid membrane is similar to that in the choroid plexus; in both tissues it is about two to four times greater than in the kidney cortex. The role of alkaline phosphatase in the calcification of bones was stressed by Robison (1932). Whether or not alkaline phosphatase has a similar role in intra-ocular calcifications is an open problem.

At physiological $p \mathrm{H}$ the 5-nucleotidase is very active, whereas the activity of the alkaline phosphatase is very low. Thus it seems possible that in physiological metabolism, as in pathological calcification, 5-nucleotidase is more important than alkaline phosphatase.

We do not know whether the phosphatases play a part in intraocular fluid formation. It seems probable that the presence of such a factor in the blood intra-ocular fluid barrier (uveal membrane), has some bearing on selective filtration. The fact that the distribution of phosphate ions does not follow Donnan's law seems to point to a special regulating mechanism.

\section{SUMMARY}

(1) The human choroid shows great alkaline phosphatase activity.

(2) A specific phosphatase, 5-nucleotidase, is present in retina and choroid.

(3) These enzymes may perhaps play a part in intra-ocular fluid formation and in intra-ocular calcifications.

I wish to express my thanks to Professor E. J. King for his advice and for the opportunity to perform the experiments in his laboratory.

\section{REFERENCES}

Concilis, N. De (1934). Sperimentale, 88, 793.

Friedenwald, J. S., and Crowell, J. E. (1949). Bull. Johns Hopkins Hosp.. 84, 568.

Gulland, J. M., and JaCKSON, E. M. (1938). Biochem. J., 32, 597.

King, E. J., and Armstrong, A. R. (1934). Canad. med. Ass. J., 31, 376.

ReIS, J. L. (1937a). Enzymologia, Amst., 2, 110. (1937b). Ibid., 2, 183.

(1940). Bull. Soc. Chim biol., 22, 36. (1951). Biochem. J. (in the press).

Robison, R. (1932). "The Significance of Phosphoric Esters in Metabolism " University Press, New York. (Oxford Univ. Press, 1933).

SüllmanN, H. (1947). Z. Vitamin-Hormon-Fermentforsch., 1, 374. and Payot, P. (1949). Ophthalmologica, Basel, 118, 345. 